\title{
Distinct patterns of primary and motile cilia in Rathke's cleft cysts and craniopharyngioma subtypes
}

\author{
Shannon Coy ${ }^{1}$, Ziming Du ${ }^{1}$, Shu-Hsien Sheu ${ }^{2}$, Terri Woo ${ }^{1}$, Fausto J Rodriguez ${ }^{3}$, \\ Mark W Kieran ${ }^{4}$ and Sandro Santagata ${ }^{1,2,5,6}$ \\ ${ }^{1}$ Department of Pathology, Brigham and Women's Hospital, Harvard Medical School, Boston, MA, USA; \\ ${ }^{2}$ Department of Pathology, Boston Children's Hospital, Harvard Medical School, Boston, MA, USA; \\ ${ }^{3}$ Department of Pathology, Johns Hopkins University, Baltimore, MD, USA; ${ }^{4}$ Department of Pediatric \\ Oncology, Dana-Farber Cancer Institute, Harvard Medical School, Boston, MA, USA; ${ }^{5}$ Department of Cancer \\ Biology, Dana-Farber Cancer Institute, Harvard Medical School, Boston, MA, USA and ${ }^{6}$ Ludwig Center at \\ Harvard, Harvard Medical School, Boston, MA, USA
}

\begin{abstract}
Cilia are highly conserved organelles, which serve critical roles in development and physiology. Motile cilia are expressed in a limited range of tissues, where they principally regulate local extracellular fluid dynamics. In contrast, primary cilia are expressed by many vertebrate cell types during interphase, and are intimately involved in the cell cycle and signal transduction. Notably, primary cilia are essential for vertebrate hedgehog pathway activity. Improved detection of motile cilia may assist in the diagnosis of some pathologic entities such as Rathke's cleft cysts, whereas characterizing primary cilia in neoplastic tissues may implicate cilia-dependent signaling pathways as critical for tumorigenesis. We show that immunohistochemistry for the nuclear transcription factor FOXJ1, a master regulator of motile ciliogenesis, robustly labels the motile ciliated epithelium of Rathke's cleft cysts. FOXJ1 expression discriminates Rathke's cleft cysts from entities in the sellar/ suprasellar region with overlapping histologic features such as craniopharyngiomas. Co-immunohistochemistry for FOXJ1 and markers that highlight motile cilia such as acetylated tubulin (TUBA4A) and the small GTPase ARL13B further enhance the ability to identify diagnostic epithelial cells. In addition to highlighting motile cilia, ARL13B immunohistochemistry also robustly highlights primary cilia in formalin-fixed paraffin-embedded sections. Primary cilia are present throughout the neoplastic epithelium of adamantinomatous craniopharyngioma, but are limited to basally oriented cells near the fibrovascular stroma in papillary craniopharyngioma. Consistent with this differing pattern of primary ciliation, adamantinomatous craniopharyngiomas express significantly higher levels of SHH, and downstream targets such as PTCH1 and GLI2, compared with papillary craniopharyngiomas. In conclusion, motile ciliated epithelium can be readily identified using immunohistochemistry for FOXJ1, TUBA4A, and ARL13B, facilitating the diagnosis of Rathke's cleft cysts. Primary cilia can be identified by ARL13B immunohistochemistry in routine pathology specimens. The widespread presence of primary cilia in adamantinomatous craniopharyngioma implicates cilia-dependent hedgehog signaling in the pathogenesis of adamantinomatous craniopharyngioma.

Modern Pathology (2016) 29, 1446-1459; doi:10.1038/modpathol.2016.153; published online 26 August 2016
\end{abstract}

Cilia were among the first organelles to be described microscopically. They are highly evolutionarily conserved, present in all of the extant eukaryotic kingdoms of life. In vertebrates, cilia are principally

Correspondence: Dr. S Santagata, MD, PhD, Division of Neuropathology, Department of Pathology, Brigham and Women's Hospital, 75 Francis Street, Amory 3, Boston, MA 02115, USA. E-mail: ssantagata@bics.bwh.harvard.edu

Received 19 May 2016; revised 13 July 2016; accepted 13 July 2016; published online 26 August 2016 characterized by a 9+2 (motile) or 9+0 (primary) axonemal microtubule structure. Motile cilia are typically present in tufts at the apical surface of cells in specialized epithelia such as the ependymal lining of the ventricular system of the brain, the fallopian tubes and the upper respiratory tracts. There they provide essential regulation of the extracellular space through modulation of fluid dynamics, mucus transport, and transportation of germ cells. In contrast, primary cilia are generally immotile and are expressed as solitary structures in 
many epithelial and mesenchymal cells. Primary cilia were described as early as 1844 by Ecker; however, it was not until recently that their essential roles in regulating diverse cellular processes were appreciated and began to be rigorously explored experimentally. ${ }^{1}$

The presence of motile cilia is frequently an informative finding in the evaluation of pathology specimens. The generation of multiple motile cilia requires de novo generation of numerous centrioles reinforcing cell cycle exit. As such, motile cilia in adult organisms are principally expressed by quiescent, terminally differentiated cells. ${ }^{2}$ The presence of multi-ciliated cells in biopsies, surgical, and cytologic specimens is accordingly invoked as evidence of benignity or of specific lineage differentiation in a variety of settings. ${ }^{3}$ The presence of motile cilia may also greatly assist in the diagnosis of specific lesions such as Rathke's cleft cysts. ${ }^{4}$ Though identification of motile cilia is often straightforward, technical factors such as crushed or distorted specimens or limited sampling may make unequivocal identification of cilia challenging. Despite such difficulties, immunohistochemistry that highlights ciliated cells is not routinely used as an adjunct to morphologic analysis.

In contrast to motile cilia, primary cilia are rarely observed or described in the evaluation of pathology specimens. Primary cilia are not readily identifiable with standard stains such as hematoxylin and eosin (H\&E). Nevertheless, primary cilia have important roles in numerous aspects of cell biology. Primary cilia are essential for hedgehog pathway activity in vertebrates. They are necessary both for production of repressor GLI transcription factor in the absence of ligand, and activation of downstream signaling in the presence of ligand. ${ }^{5}$ Numerous other critical signaling pathways such as those for Wnt/ $\beta$-catenin, receptor tyrosine kinase, and Notch signaling are also intrinsically regulated by primary cilia in a variety of contexts. ${ }^{6-8}$ Primary cilia have long been thought to function as calcium responsive mechanosensors, ${ }^{9}$ though recent findings have cast doubt on this hypothesis. ${ }^{10}$ Owing to the dual function of the mother centriole as both the basal body of the primary cilia in interphase and a central component of the centrosomal spindle pole in mitosis, primary cilia are intrinsically involved in cell cycle progression. ${ }^{11}$ A growing number of ciliopathy syndromes have been described, with pleiotropic and profound effects on development and adult physiology, illustrating the importance of primary cilia in normal human physiology. ${ }^{12}$ Despite these manifold functions, primary cilia are poorly characterized in human pathology and are not assessed during the diagnostic evaluation of surgical resection specimens.

Primary cilia are absent or greatly attenuated in a variety of cancers in a proliferation-independent manner, ${ }^{13}$ and this loss often occurs early in oncogenesis. $^{14}$ However, neoplasms driven by oncogenic activation of the hedgehog pathway, including basal cell carcinoma and a subset of medulloblastomas, frequently retain primary cilia likely reflecting the dependence of upstream hedgehog signaling on this organelle. Retention of primary cilia has also been observed in lesions with activating Wnt/ $\beta$-catenin pathway mutations, an observation whose basis is less clear, but which may reflect direct regulation of $\mathrm{Wnt} / \beta$-catenin signaling by cilia. ${ }^{6}$ Nevertheless, the status of primary cilia in a variety of neoplasms is unknown.

Craniopharyngiomas-rare epithelial neoplasms of the sellar/suprasellar region-provide one such example. The craniopharyngioma family consists of two entities: adamantinomatous craniopharyngioma, which occur both in children and adults, and papillary craniopharyngioma, which occur almost exclusively in adults. ${ }^{15-18}$ Adamantinomatous craniopharyngiomas are composed of basaloid sheets and cords of cells resembling adamantinomatous odontogenic epithelium with associated findings such as 'wet keratin.' Molecular analyses of adamantinomatous craniopharyngiomas demonstrate recurrent activating mutations in CTNNB1, the gene encoding $\beta$-catenin. In contrast, papillary craniopharyngiomas demonstrate a well-differentiated squamous morphology with neoplastic cells emanating from fibrovascular cores. Nearly all papillary craniopharyngiomas harbor oncogenic BRAF V600E mutations. ${ }^{15,19-23}$ Adamantinomatous craniopharyngiomas also demonstrate evidence of active hedgehog signaling with expression of PTCH1, $S H H$, and GLI. ${ }^{24-26}$ However, hedgehog responsiveness may be regionalized or limited by local expression of primary cilia, even when pathway components are highly expressed, as may occur in pancreatic ductal adenocarcinoma. ${ }^{27-29}$ Therefore, characterization of the distribution of primary cilia is critical to understanding hedgehog signaling, and possibly others such as Wnt/ $\beta$-catenin, in both cancer and normal biology.

Diagnosis of lesions of the sellar/suprasellar compartment, such as craniopharyngioma, is often challenging, owing to the small amount of lesional tissue frequently obtained in biopsies and small cyst resections, distortion of specimens, and histologic and clinical overlap between lesions. Rathke's cleft cysts can be confused with papillary craniopharyngiomas, particularly when the Rathke's cleft cyst epithelium includes regions of stratified squamous epithelium, which resembles the neoplastic epithelium of papillary craniopharyngioma. ${ }^{22,30}$ These Rathke's cleft cyst specimens often still maintain focal patches of characteristic motile ciliated epithelium, which are frequently challenging to unequivocally identify.

Although the diagnosis of adamantinomatous craniopharyngioma may be corroborated with immunohistochemistry for $\beta$-catenin, the recent development of a specific antibody for the BRAF V600E mutant protein provides pathologists with an 
additional ancillary diagnostic tool to differentiate papillary craniopharyngioma. ${ }^{20}$ Patients with recurrent BRAF mutant papillary craniopharyngioma can then be managed with targeted therapeutics with clinical benefit. ${ }^{31,32}$ While the BRAF V600E antibody allows pathologists to positively identify papillary craniopharyngioma, the evaluation of sellar/suprasellar lesions would be further facilitated by immunostains that can be used to positively support the diagnosis of Rathke's cleft cyst. ${ }^{33}$ Reactivity for cytokeratins such as CK8 and CK20 has been suggested as one option for differentiating Rathke's cleft cyst and craniopharyngioma, ${ }^{34,35}$ but variability in this staining pattern has been reported. ${ }^{36}$

In light of the finding of Shin et $a l^{4}$ that the most distinctive features of Rathke's cleft cysts are columnar or cuboidal epithelium and ciliation, we reasoned that an immunohistochemical method to identify ciliated epithelium would be useful for positively identifying Rathke's cleft cysts. FOXJ1 is the human homolog of the mouse Hnf-3/Hfh-4 gene and is a highly conserved member of the forkhead family of transcription factors. FOXJ1 and its homologs are critical regulators of motile ciliogenesis in diverse species from Drosophila melanogaster to man. ${ }^{37}$ We hypothesized that immunohistochemical staining for nuclear localization of the FOXJ1 transcription factor would allow for the robust identification of regions of ciliated epithelium, and that such a marker could be used in conjunction with markers such as acetylated tubulin and ARL13B, ${ }^{38}$ which highlight both motile and primary cilia. Development of such immunohistochemical methods would also enable the characterization of primary cilia in sellar/suprasellar lesions, which might be used to suggest novel therapeutic approaches, given the oft-observed correlation between active hedgehog signaling and presence of primary cilia in neoplastic disease.

\section{Materials and methods}

\section{Human Tissue Characterization}

Formalin-fixed and paraffin-embedded tissues from 16 Rathke's cleft cysts, 23 papillary craniopharyngiomas, and 19 adamantinomatous craniopharyngioma cases, including paired primary-recurrence resections in one papillary craniopharyngioma and two adamantinomatous craniopharyngiomas, were identified and retrieved from the archives of the Department of Pathology, Brigham and Women's Hospital and Boston Children's Hospital in accordance with institutional review board (IRB) approval. The characteristics of all the cases are listed in Supplementary Table 1. The cases have a wide age range with similar demographic characteristics. All craniopharyngioma and Rathke's cleft cyst cases were retrospectively reviewed in accordance with the revised WHO 2007 classification of brain tumors by the authors (SC and SS). Targeted genotyping was available for 18 of the papillary craniopharyngioma cases, and showed that all the cases harbored a BRAF V600E mutation, which was also confirmed by mutation-specific immunohistochemistry (in all the 18 sequenced cases, and 20 cases in total). Genotyping of papillary craniopharyngioma demonstrated absence of CTNNB1 mutations in all the cases examined (18/18). CTNNB1 immunohistochemistry was available for 20 papillary craniopharyngioma cases, $100 \%$ of which showed a membranous (inactive) staining pattern. Targeted genotyping data were available for 15 of the adamantinomatous craniopharyngioma cases in this series, and showed that $12 / 15(80 \%)$ cases harbored exonic CTNNB1 mutations. CTNNB1 immunohistochemistry was available for 16 cases, all of which showed characteristic cytoplasmic and nuclear staining, including two of the three cases that were negative by sequencing. The third case did not have immunohistochemistry available. BRAF V600E mutations were not present by genotyping in any adamantinomatous craniopharyngioma case examined (0/14). Focal BRAF V600E immunohistochemistry signal was present in one adamantinomatous craniopharyngioma case $(1 / 15,7 \%)$, though this case was negative by genotyping. Whole-exome sequencing demonstrates no mutations in SHH, PTCH, GLI, $S U F U$, or $S M O$ in any adamantinomatous craniopharyngioma or papillary craniopharyngioma case. ${ }^{15}$

\section{Immunohistochemistry}

FOXJ1 immunohistochemistry. Staining was performed as previously reported. ${ }^{39}$ In brief, tissue sections were cut at $4 \mu \mathrm{m}$ and deparaffinized in xylene and absolute alcohol. Endogenous peroxidase activities were blocked with a solution of $3 \%$ hydrogen peroxide and absolute alcohol 1:1 for $15 \mathrm{~min}$. Heat-induced antigen retrieval was performed with a pressure cooker $\left(122 \pm 2{ }^{\circ} \mathrm{C}\right)$ for $45 \mathrm{~s}$ at $15 \pm 5$ PSI in citrate buffer ( $\mathrm{pH}$ 6.0). Immunohistochemistry was performed using a polyclonal rabbit antibody to FOXJ1 (HPA005714, Sigma-Aldrich) at a dilution of 1:75 incubated for $45 \mathrm{~min}$ at room temperature followed by labeled polymer-HRP antirabbit IgG incubated 30 min and visualized with 3,3'diaminobenzidine (DAB; brown in color; Dako Envision System). The slides were counter-stained with hematoxylin. Images were acquired using an Olympus BX41 microscope and an Olympus DP26 digital camera.

Double-stain FOXJ1/ARL13B immunohistochemistry. Tissue preparation and FOXJ1 staining were performed as described above. Then mouse monoclonal antibody to ARL13B (75-287, clone N295B/66, UC Davis/NIH NeuroMab Facility, Davis, CA, USA) was applied at a dilution of 1:400 and incubated $45 \mathrm{~min}$ at room temperature, followed by a $30 \mathrm{~min}$ 
incubation with Poly-AP anti-mouse IgG (PV6110, Leica Biosystems, UK) at room temperature and visualized using ImmPACT Vector Red Alkaline Phosphatase (SK-5105, Vector Laboratory, CA, USA) for $15 \mathrm{~min}$. The slides were counter-stained with hematoxylin. The percentage of ciliated cells was estimated by counting primary cilia in 40 contiguous cells using a $40 \times$ objective, with counts compared for consistency in two to three regions of each section when not limited by small tissue samples. Accurate counting often requires scanning through different focal planes in the $z$ axis given the small size of cilia.

Double-stain FOXJ1/TUBA4A (acetyl K40) immunohistochemistry. Tissue preparation and FOXJ1 staining were performed as described above. Then mouse monoclonal antibody to TUBA4A (acetyl K40, clone 6-11 B-1; ab11323, Abcam) was applied at a dilution of 1:20000 and incubated $45 \mathrm{~min}$ at room temperature, followed by Poly-AP anti-mouse IgG (PV6110, Leica Biosystems, UK) incubation of $30 \mathrm{~min}$ at room temperature and visualized using ImmPACT Vector Red Alkaline Phosphatase (SK-5105, Vector Laboratory, CA, USA) for $10 \mathrm{~min}$. The slides were counter-stained with hematoxylin.

Beta-catenin immunohistochemistry. Tissue preparation was performed as above. Beta-catenin immunohistochemistry was performed using a mouse monoclonal antibody (BD Biosciences, Cat \#610154, clone: 14). Antigen retrieval was performed using a pressure cooker in citrate buffer $(\mathrm{pH}=6.0$, 1:1000 dilution) with a $45 \mathrm{~min}$ incubation followed by Dako anti-mouse-HRP for $30 \mathrm{~min}$ at room temperature.

\section{RNAscope}

RNAscope was performed to detect expression of mRNA encoding components of the hedgehog signaling pathway in formalin-fixed paraffinembedded sections (Supplementary Table 1). The protocol was performed according to the manufacturer's recommendations using Probe-Hs-GLI2 (Custom design, Advanced Cell Diagnostics, USA), Probe-Hs-SHH (Cat \#600951, Advanced Cell Diagnostics, USA), and Probe-Hs-PTCH1 (Cat \#405781 Advanced Cell Diagnostics, USA). The Probe-HsPPIB (Cat \#313901, Advanced Cell Diagnostics, USA) was used as a positive control. The probes were visualized with $\mathrm{DAB}$ and cell nuclei were stained with hematoxylin. Slides were digitally scanned, and evaluated by two pathologists (ZD and SC) and then analyzed using open-source CellProfiler image analysis software (http://www.cellprofiler.org/). ${ }^{40}$

\section{Results}

We recently reported that FOXJ1 is expressed in the nucleus of ciliated ependymal cells that line the ventricle of the human brain, as well as in welldifferentiated ependymoma and choroid plexus tumors. ${ }^{39,41}$ Using an immunohistochemistry protocol developed for that study, we analyzed the expression of FOXJ1 protein in a panel of 16 Rathke's cleft cysts (Supplementary Table 1; Figure 1a). We observed strong nuclear staining for FOXJ1 (Figure 1b) in 14/16 Rathke's cleft cysts (88\%). Nuclear FOXJ1 staining was also present in fallopian tube controls (Figure 1c). FOXJ1 was expressed in $10 / 10(100 \%)$ Rathke's cleft cyst cases in which motile cilia were evident by H\&E. In these cases, FOXJ1 was expressed in approximately $70 \%$ of surface epithelial cells, reflecting the presence of both ciliated cells and intercalated non-ciliated secretory cells. The proportion of FOXJ1-positive cells was reduced in a subset of Rathke's cleft cyst cases $(6 / 16 ; 38 \%)$, predominantly in cases in which the epithelium was less abundant and was generally cuboidal in morphology. The two Rathke's cleft cysts that were entirely negative for FOXJ1 lacked any apparent ciliated epithelium on H\&E, demonstrating low cuboidal epithelium with prominent regions that were flattened or had focal stratified squamous epithelium. Although ciliation is the most specific histologic feature of Rathke's cleft cysts, it is not uniformly present, ${ }^{4}$ and a diagnosis of Rathke's cleft cyst can be made in the absence of ciliation in the appropriate clinicopathologic context. FOXJ1 staining was also robustly expressed in cases with severely disrupted architecture (Figure 1d), in which the presence of cilia could not be readily confirmed.

To confirm the presence of motile cilia and more rigorously assess the relationship between nuclear FOXJ1 expression and ciliation, we optimized immunohistochemistry protocols for markers of mature motile cilia. Tubulin alpha 4 a (TUBA4A) is a ubiquitously expressed member of the tubulin super-family of proteins, which has its highest levels of expression in the brain. ${ }^{42}$ Acetylation of tubulin alpha promotes the assembly and stabilization of axonemal microtubules within cilia, and immunofluorescence for acetylated tubulin has been previously described for the study of cilia. ${ }^{38} \mathrm{We}$ performed immunohistochemistry for acetylated TUBA4A on sections of fallopian tube and observed strong apical expression on the surface of numerous cells, whereas scattered cells lacked surface staining (Figure 2a). This pattern of staining is consistent with the presence of ciliated epithelial cells in the fallopian tube separated by intercalated, nonciliated secretory cells.

In Rathke's cleft cysts with well-preserved ciliated epithelium, we observed strong immunostaining for TUBA4A in a continuous pattern on the apical surface of the epithelial lining (Figure $2 \mathrm{~b}$ ). We also frequently noted staining for TUBA4A in the cytoplasm of the stromal cells that comprise the cyst wall and of the normal parenchymal cells of the adenohypophysis, which are often resected along with the Rathke's cleft cyst (Figure 2c). The presence 
of strong cytoplasmic staining in non-ciliated cells confounds the use of acetylated tubulin as a single marker of motile ciliation, particularly in cases that have disrupted architecture (Figure 2d).

Accordingly, we developed co-immunohistochemistry for FOXJ1 and TUBA4A, expecting that a double stain might allow for more definitive identification of ciliated epithelium. Co-staining for TUBA4A and FOXJ1 demonstrated a robust and specific concordance between areas of TUBA4A (red) and nuclear FOXJ1 (brown) expression in the fallopian tube (Figure 3a). Ciliated cells strongly expressed both markers, whereas the intercalated non-ciliated secretory cells were negative for both markers.

Similarly, double staining for TUBA4A and FOXJ1 demonstrated strong uniform staining in Rathke's cleft cysts that had well-developed and wellpreserved ciliated epithelium (Figure 3b). In 13/14 $(93 \%)$ Rathke's cleft cyst cases that had FOXJ1 expression, ciliation was confirmed by TUB4A staining and limited to regions of nuclear FOXJ1 expression. The single discordant case displayed only very scant epithelium with few FOXJ1-positive cells, and the absence of obvious cilial staining could have represented sampling error due to cilia outside the plane of the section in the limited specimen. In Rathke's cleft cysts with fragmented epithelium, which had been difficult to classify by conventional H\&E histology, co-staining with FOXJ1 and TUBA4A supported the presence of ciliated epithelium (Figure 3c). Six Rathke's cleft cysts (38\%) displayed significant disruption of the cyst architecture or only isolated clusters and strips of epithelial cells without clear cilia on H\&E. Immunohistochemistry subsequently revealed strong nuclear FOXJ1 expression and TUBA4A positive motile cilia in epithelial cells in four of these ambiguous cases (66\%), supporting the diagnosis of Rathke's cleft cyst. Regions of flattened epithelium, which may represent early squamous metaplasia or atrophic change were present at least focally in $14 / 16$ cases (88\%), and were FOXJ1 and TUBA4A negative (Supplementary Figure 1, arrows). Stratified squamous epithelium
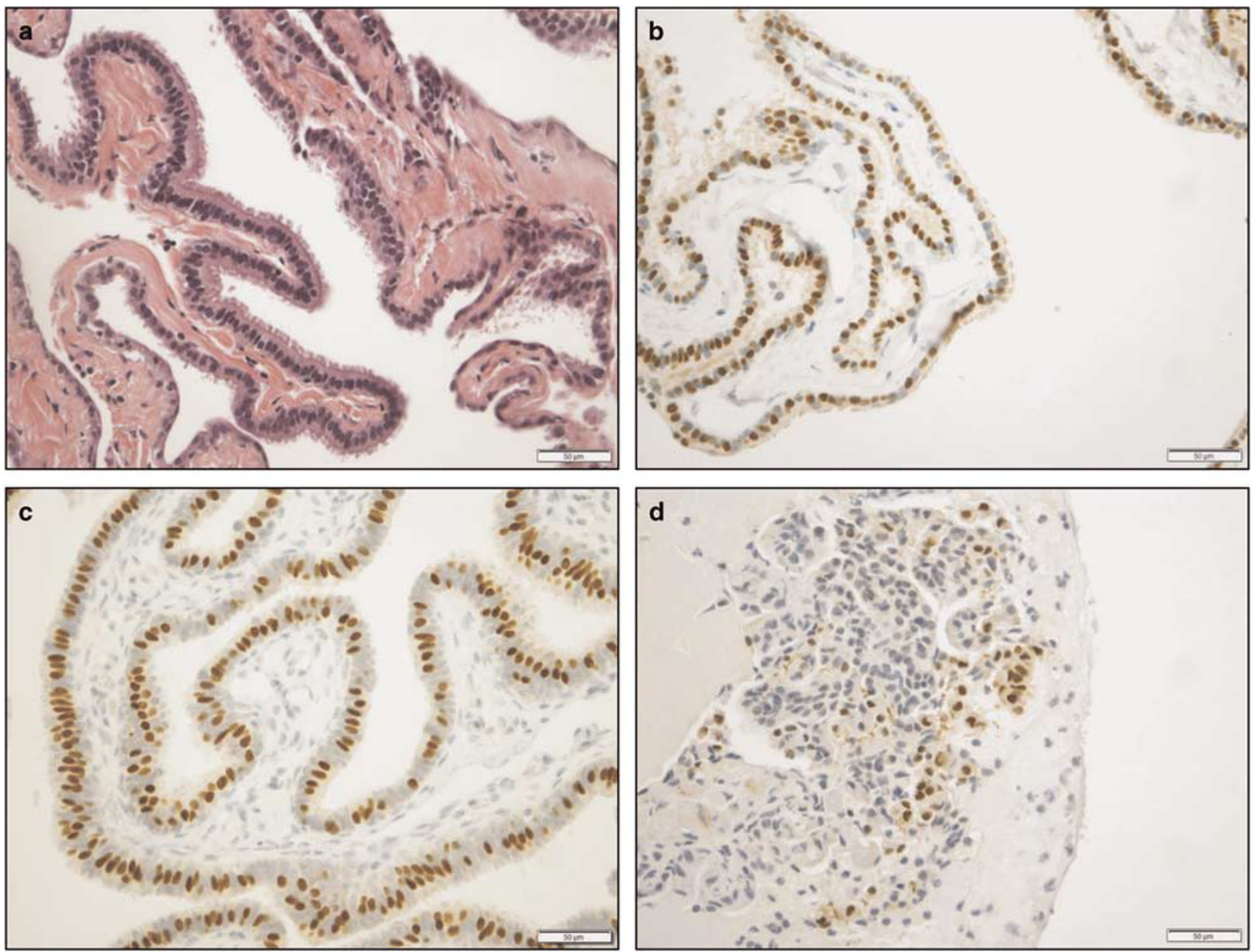

Figure 1 Hematoxylin and eosin (H\&E) stained section of Rathke's cleft cyst (a). FOXJ1 immunohistochemistry (brown) of Rathke's cleft cyst (b and d) and fallopian tube (c). (d) Shows immunohistochemistry on a Rathke's cleft cyst that has significantly disrupted and fragmented cyst epithelium. Staining demonstrates robust nuclear expression of FOXJ1 in multi-ciliated epithelial cells. Scale bars, $50 \mu \mathrm{m}$. 
was focally present in 4/16 cases (25\%), and was negative for both FOXJ1 and TUBA4A (Figure 3d).

Given the potentially confounding cytoplasmic staining observed with TUBA4A, we validated our findings with an additional marker of cilia, ADPRibosylation Factor-Like 13B (ARL13B), a small GTPase that is a critical promoter of ciliogenesis. ARL13B localizes to the cilia and has important roles in the production and maintenance of both primary and motile cilia. Supporting its fundamental role in ciliogenesis, mutations in the $A R L 13 B$ gene result in a ciliopathy-Joubert Syndrome $8 .^{43}$

We performed immunohistochemistry on a subset of our Rathke's cleft cyst cases with an antibody to ARL13B. Similar to the pattern of staining observed with TUBA4A, ARL13B immunohistochemistry demonstrated robust staining of the apical surface of ciliated cells of both the fallopian tube (Figure 4a) and of the epithelium of Rathke's cleft cysts (Figure 4b). Co-staining revealed strong apical ARL13B staining of motile cilia on cells that also expressed nuclear FOXJ1, both in the fallopian tube (Figure 4c) and in Rathke's cleft cysts (Figure 4d).

As previously reported, ${ }^{38}$ ARL13B is also highly expressed in primary cilia. ARL13B immunohistochemistry revealed the presence of numerous subcellular structures consistent with primary cilia. Scattered primary cilia were observed in Rathke's cleft cyst epithelial cells $(25 \pm 13 \%$ of cells; Supplementary Figure 2a, arrows), though this may be an underestimation. The small size of primary cilia (approximately $500 \mathrm{~nm}$ in diameter) coupled with the limited spatial representation in $5 \mu \mathrm{m}$ formalin-fixed paraffin-embedded sections of cells that are up to $10 \mu \mathrm{m}$ in diameter likely results in a proportion of cilia lying outside the plane of the section. Primary cilia are also present in anterior pituitary cells (Supplementary Figure 2b, arrows), and stromal cells (not pictured) in nearly all cases of Rathke's cleft cyst and craniopharyngioma examined (96\%), which serves as an excellent internal positive control for staining. The two Rathke's cleft cyst cases
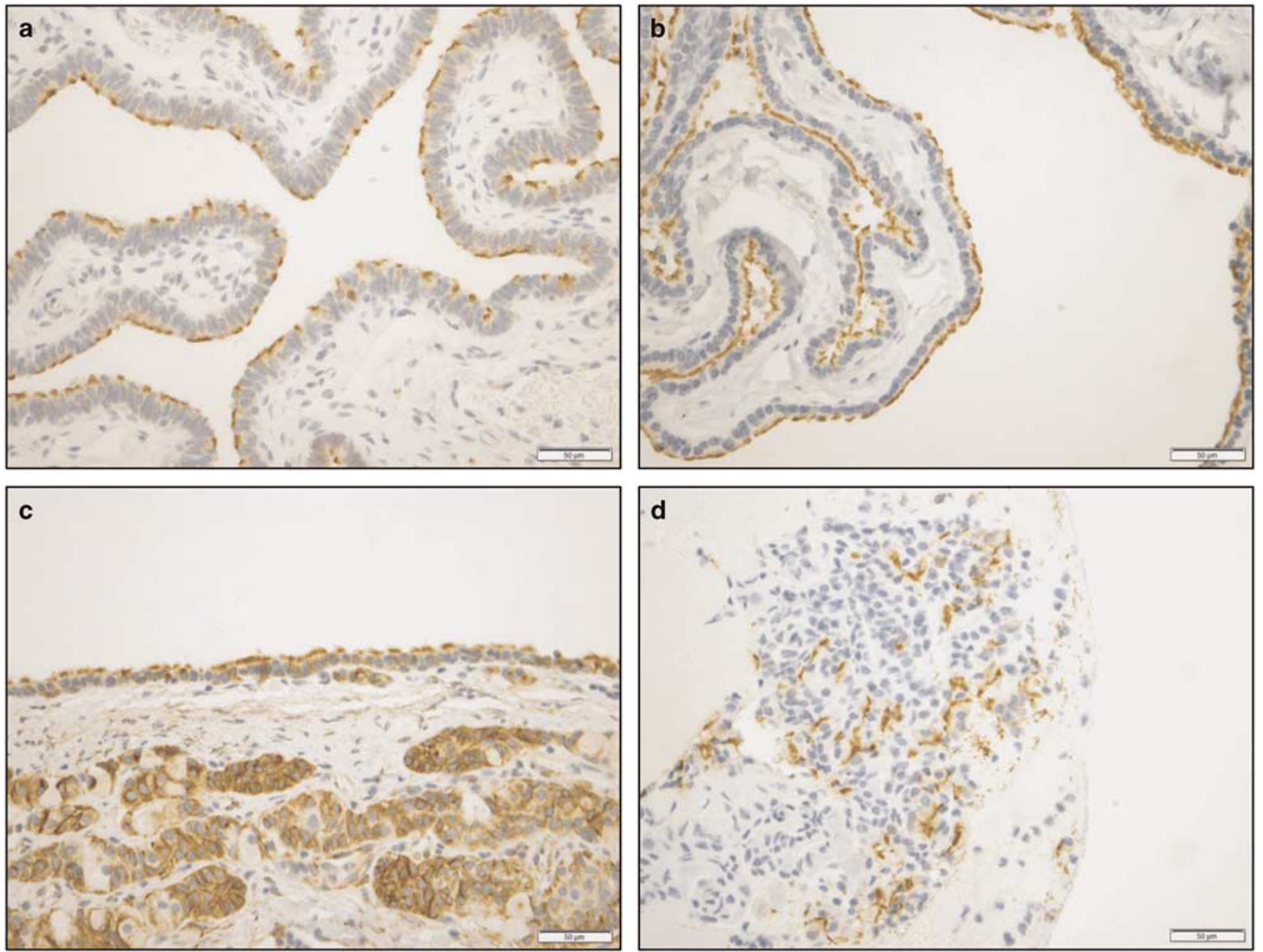

Figure 2 Immunohistochemistry for acetylated TUBA4A (brown) on fallopian tube (a) Rathke's cleft cyst (b), Rathke's cleft cyst with underlying clusters of normal anterior pituitary cells (c), and Rathke's cleft cyst that has significantly disrupted and fragmented cyst epithelium (d). Immunohistochemistry for TUBA4A demonstrates robust staining of cilia; however, there is significant cytoplasmic background particularly in anterior pituitary cells. Scale bars, $50 \mu \mathrm{m}$. 

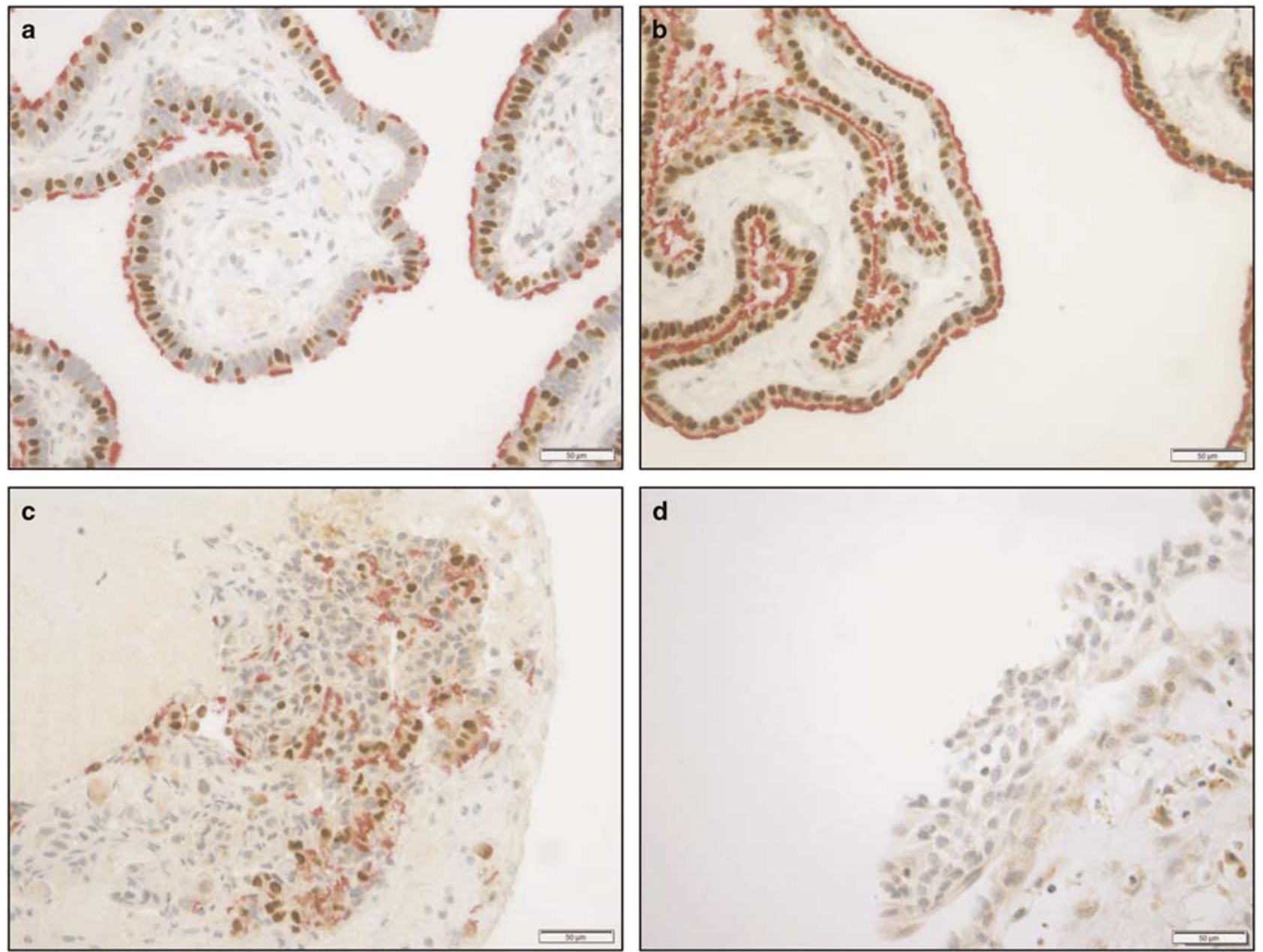

Figure 3 Co-immunohistochemistry for both FOXJ1 (brown) and acetylated TUBA4A (red) on fallopian tube (a), Rathke's cleft cyst (b), Rathke's cleft cyst that has significantly disrupted and fragmented cyst epithelium (c), and Rathke's cleft cyst with stratified squamous epithelium (d). Co-immunohistochemistry shows concordance between FOXJ1 and TUBA4A. Scale bars, $50 \mu \mathrm{m}$.

with no identifiable stromal cilia demonstrated total obliteration of the stroma by inflammatory cells and degradative changes. Notably, in contrast to motile cilia, which were always localized to regions of nuclear FOXJ1 expression, primary cilia showed no correlation with FOXJ1 expression. ARL13B showed essentially identical results to TUBA4A with regard to motile cilia staining in all Rathke's cleft cyst cases examined, but showed much greater specificity for both motile and primary cilia while generating far less cytoplasmic background staining. We therefore used ARL13B immunohistochemistry to label both primary and motile cilia in all of our subsequent experiments.

We next evaluated FOXJ1 expression in 19 cases of adamantinomatous craniopharyngioma and 23 cases of papillary craniopharyngioma. The neoplastic epithelial cells of adamantinomatous craniopharyngioma (Figure 5a) were uniformly $(19 / 19,100 \%)$ negative for FOXJ1 (Figure 5b). The vast majority of papillary craniopharyngiomas (Figure 5c) were also completely negative for FOXJ1 (21/23, 91\%; Figure 5d). However, focal FOXJ1-positive cells with
ARL13B positive motile cilia were present in 2 of 23 cases $(9 \%)$. In both of these papillary craniopharyngioma cases, FOXJ1-positive motile ciliated cells were present at the apical surface of the squamous epithelium (Figure 5e). Rare FOXJ1-positive cells deeper in the epithelium were occasionally observed that appeared to be non-ciliated, which likely results from cilia lying outside of the plane of section (arrow). Rare goblet cells were also present (not pictured). Interestingly, strips of FOXJ1-positive motile ciliated columnar epithelium reminiscent of Rathke's cleft cyst epithelium were also present in these two papillary craniopharyngioma cases (Figure 5f). These two cases presumably represent 'ciliated craniopharyngiomas,' that have been reported, but only rarely. ${ }^{44}$ Notably, these two 'ciliated craniopharyngioma' cases were both, as would be expected for papillary craniopharyngioma, positive for BRAF V600E by genotyping and immunohistochemistry (Supplementary Figure 3). The apical ciliated cells appeared to also be positive for BRAF V600E by immunohistochemistry as did the surrounding squamous cells, though off-target 

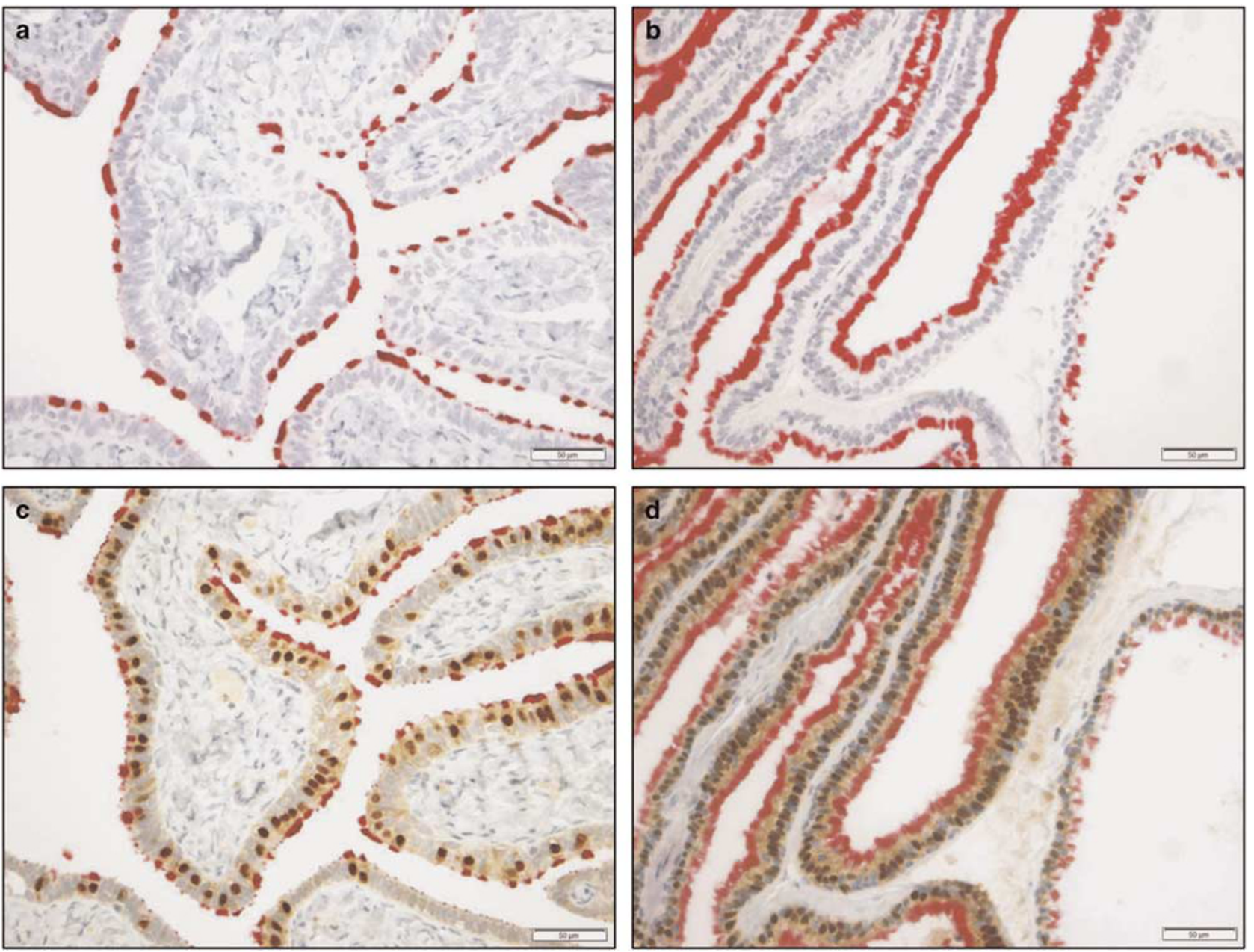

Figure 4 Immunohistochemistry for ARL13B on fallopian tube (a) and Rathke's cleft cyst (b). Co-immunohistochemistry for FOXJ1 (brown) and ARL13B (red) on fallopian tube (c) and Rathke's cleft cyst (d). ARL13B demonstrates robust staining of motile cilia, and colocalization with FOXJ1 in motile ciliated cells. There is minimal background staining with ARL13B immunohistochemistry. Scale bars, $50 \mu \mathrm{m}$.

axonemal dynein staining may confound this observation. ${ }^{30}$

In contrast to the limited expression of nuclear FOXJ1 in craniopharyngioma, ARL13B staining revealed numerous primary cilia in both adamantinomatous craniopharyngioma (Figure 6a) and papillary craniopharyngioma (Figure 6b). These structures were present in the epithelium of all the cases that we examined. Epithelial primary cilia were limited to the basally oriented tumor cells that surround fibrovascular cores in papillary craniopharyngioma. Primary cilia were present in the first two to three cell layers, in all the papillary craniopharyngioma cases, in $69 \pm 12 \%$ of cells. The non-basal 'apical' cells ( $>3$ layers away from fibrovascular cores in the $x-y$ plane) were negative for primary cilia ( $<1 \%$ of cells). The two 'ciliated craniopharyngioma' cases also expressed primary cilia in the neoplastic squamous cells in a predominantly basally oriented distribution, similar to the other papillary craniopharyngiomas (Figure 5e, arrowhead). However, occasionally both the FOXJ1- positive (Supplementary Figure 4a) and FOXJ1negative (Supplementary Figure 4b) apical cells in these cases displayed solitary cilia, which may represent primary cilia. In contrast, adamantinomatous craniopharyngiomas diffusely expressed cilia in both the palisading basally oriented cells $(90 \pm 6 \%$ of cells), as well as the non-palisading 'apical' cells ( $>2$ layers from palisading layer in the $x-y$ plane, $82 \pm 5 \%$ of cells) in 19/19 (100\%) cases (Figure 6c).

Previous reports showed that adamantinomatous craniopharyngiomas express essential components of the hedgehog signaling pathway, with elevated SHH expression detected by RT-PCR. By immunohistochemistry, downstream targets of hedgehog signaling appear to be enriched in cells with nuclear $\beta$-catenin, which are often present in focal whorls and near regions of 'ghost cells'. ${ }^{24,25,35}$ Given the dependence of hedgehog signaling on primary cilia, we hypothesized that primary cilia might co-localize with regions of active hedgehog signaling. Using RNAscope in situ hybridization, we found that SHH mRNA expression was negligible in papillary 

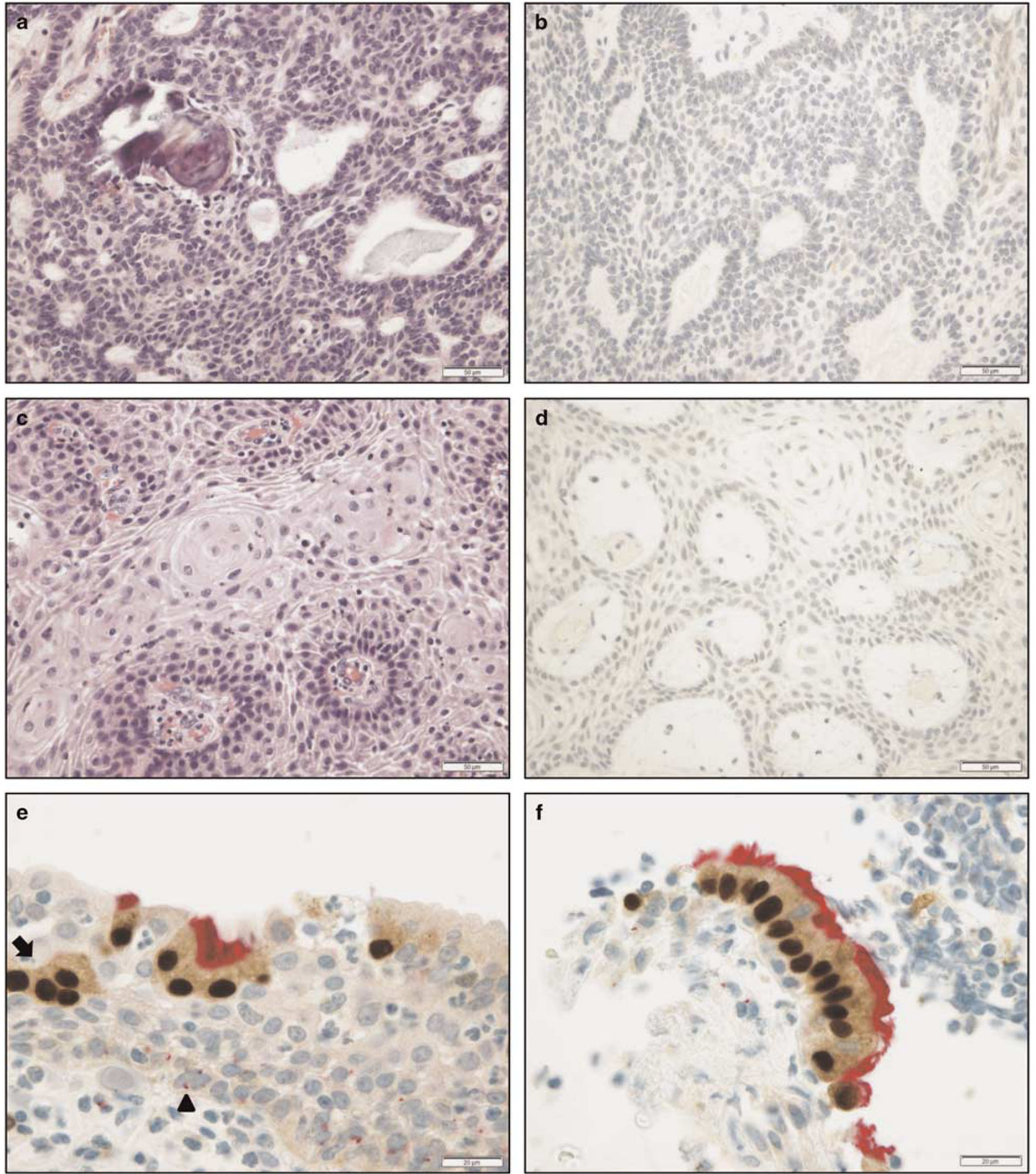

Figure 5 Hematoxylin and eosin (H\&E) stained section of adamantinomatous craniopharyngioma (a) and papillary craniopharyngioma (c). FOXJ1 immunohistochemistry (brown) for adamantinomatous craniopharyngioma (b) and papillary craniopharyngioma (d). Coimmunohistochemistry for FOXJ1 (brown) and ARL13B (red) on 'ciliated papillary craniopharyngioma' (e and f). Adamantinomatous craniopharyngiomas do not express FOXJ1. The vast majority of papillary craniopharyngioma do not express FOXJ1; however, rare ciliated BRAF V600E positive papillary craniopharyngioma cases show FOXJ1 and ARL13B expression with apical multi-ciliated cells. In (e) the arrow highlights FOXJ1 nuclear positive cells in deeper layers and the arrowhead highlights primary cilia in basal cells. Scale bars, either $50 \mu \mathrm{m}(\mathbf{a}-\mathbf{d})$ or $20 \mu \mathrm{m}$ (e and $\mathbf{f})$. 

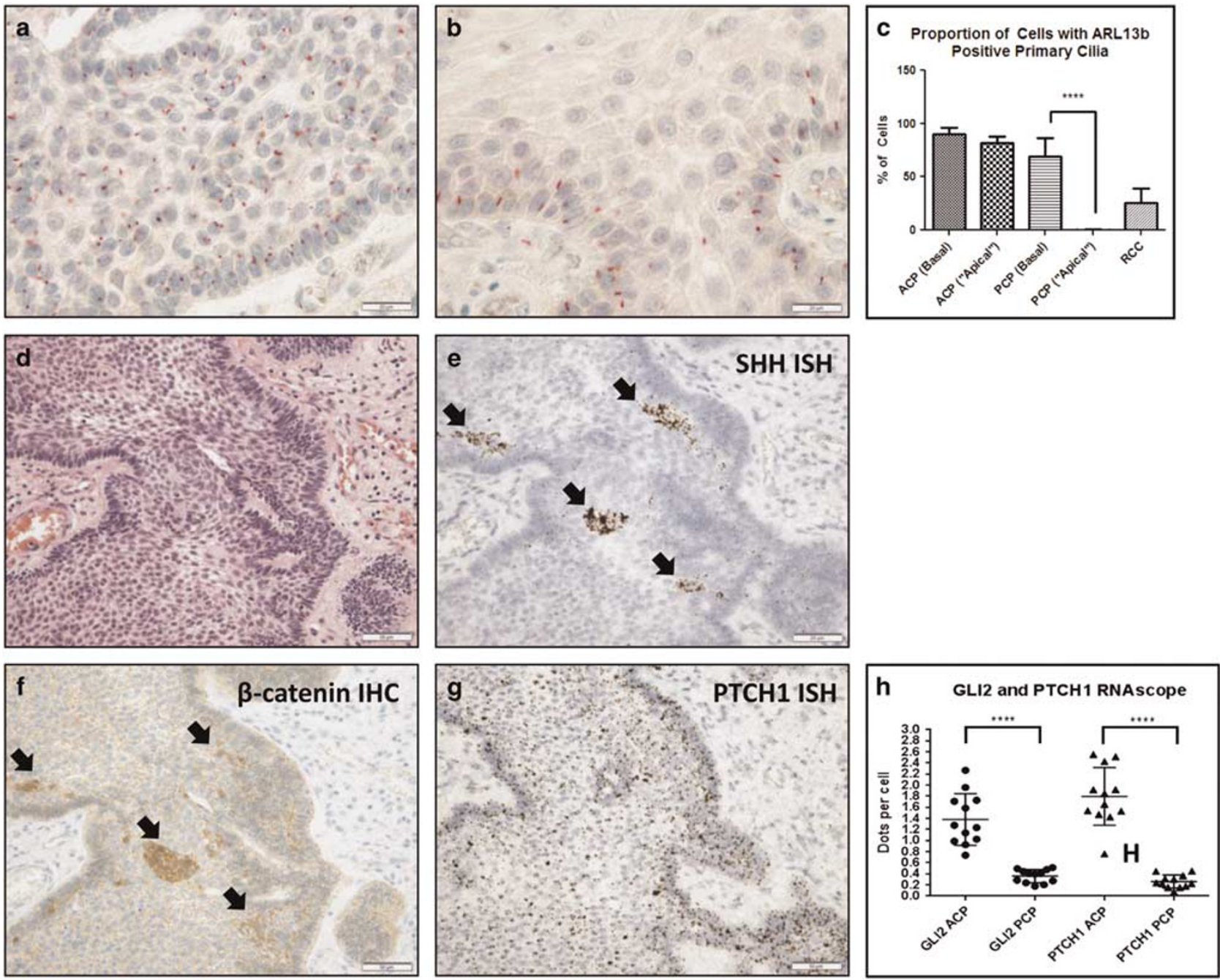

Figure 6 ARL13B immunohistochemistry (red) on adamantinomatous craniopharyngioma (a) and papillary craniopharyngioma (b). Adamantinomatous craniopharyngiomas diffusely express primary cilia, in both the palisading basal cells and non-palisading 'apical' cells in $100 \%$ of cases. Papillary craniopharyngiomas express primary cilia in basally oriented cells near fibrovascular cores in $100 \%$ of cases, but show no expression of primary cilia in more 'apical' cells. Statistical analysis of primary cilia in different lesions (c). Differences in expression between 'apical' and 'basal' cells are statistically significant $(* * * * P<0.05)$. Matched serial sections of an adamantinomatous craniopharyngioma with hematoxylin and eosin (H\&E) (d), RNAscope in situ hybridization for SHH (e) or PTCH1 (g) mRNA, or immunohistochemistry for $\beta$-catenin (f). Regions of high SHH mRNA expression (e, arrows) show excellent correlation with regions of nuclear/cytoplasmic $\beta$-catenin translocation (f, arrows). Statistical analysis of PTCH1 and GLI2 expression in adamantinomatous craniopharyngioma and papillary craniopharyngioma (h). Differences in expression are statistically significant $\left({ }^{* * * *} P<0.05\right)$. Scale bars, $20 \mu \mathrm{m}$ (a and b) or $50 \mu \mathrm{m}(\mathbf{d}-\mathbf{g})$.

craniopharyngioma (Supplementary Figure 5d), similar to the findings from recent transcriptomic analysis. ${ }^{45}$ However, we found discrete clusters of cells in adamantinomatous craniopharyngioma (Figure 6d) that strongly expressed $\mathrm{SHH}$ mRNA (Figure 6e) with striking nearly perfect colocalization of the SHH mRNA signal with clusters of cells that had nuclear/cytoplasmic translocation of $\beta$-catenin protein (Figure 6f). We found that expression of mRNA transcripts that serve as markers of hedgehog pathway activation such as PTCH1 (Figure 6g) and GLI2 (Supplementary Figure 5a) were expressed at significantly higher levels in adamantinomatous craniopharyngioma (Figure 6h) than in papillary craniopharyngioma (Supplementary Figure 5b, c). These transcripts were diffusely expressed across the epithelium of adamantinomatous craniopharyngioma, in a similar pattern to the widespread presence of primary cilia on the epithelial cells of adamantinomatous craniopharyngioma. These findings suggest that there is diffuse ciliadependent hedgehog pathway activation across the epithelium of adamantinomatous craniopharyngioma, and that hedgehog signaling is not solely localized to the clusters of cells that express $S H H$ mRNA and have nuclear/cytoplasmic $\beta$-catenin translocation. 


\section{Discussion}

Here, we show that immunohistochemistry for critical components of cellular ciliogenesis programs including a nuclear transcriptional regulator of motile ciliogenesis, FOXJ1, and markers of primary and motile cilia-acetylated tubulin (TUBA4A) and ARL13B - may be used to facilitate characterization of cilia and improve diagnostic accuracy in sellar/ suprasellar lesions. FOXJ1 robustly and specifically labels the motile ciliated cells of Rathke's cleft cysts, and is present in a large majority of cases (88\%). In contrast, epithelial FOXJ1 staining is not observed in adamantinomatous craniopharyngioma $(0 \%$ of cases), and is only rarely observed very focally in papillary craniopharyngioma $(9 \%)$. We also show a strong concordance between the expression of FOXJ1 and the presence of motile cilia, confirmed with two markers of cilia: acetylated tubulin (TUBA4A) and ARL13B. Given the near-perfect concordance of FOXJ1 expression and the presence of motile cilia in Rathke's cleft cysts, fallopian tube epithelium, and other cell types, FOXJ1 immunohistochemistry is useful as a general nuclear marker of motile ciliated epithelial cells. However, double staining may occasionally be useful to confirm the presence of motile cilia. Illustrating the usefulness of FOXJ1 and cilial immunohistochemical stains, 66\% of cases in our series that did not show readily detectable motile cilia on H\&E had easily recognizable motile cilia when examined with our immunohistochemical markers.

Previous studies have infrequently reported the identification of 'ciliated papillary craniopharyngioma' with apical tufts of cilia on neoplastic epithelium. ${ }^{44}$ We observed two such cases in our cohort, and demonstrated that cells at the apical surface of the neoplastic squamous epithelium stain positively for FOXJ1 and ARL13B. In one patient (Supplementary Table 1; case P11), this phenotype was present in both the primary resection and in a repeat resection 2 years later. Retrospective review of a prior pituitary adenoma resection from this patient also revealed the presence of squamocolumnar epithelium adjacent to pituitary with apical tufted cilia, demonstrating that ciliated differentiation was present early in the history of this lesion, and was preserved as the lesion progressed and recurred multiple times over a period of 6 years. Interestingly, in both FOXJ1-positive papillary craniopharyngioma cases, we observe small focal regions of ciliated FOXJ1-positive cuboidal epithelium reminiscent of Rathke's cleft cyst epithelium adjacent to, or in continuity with, the neoplastic squamous epithelium. This observation may provide additional evidence for the hypothesis that papillary craniopharyngioma and Rathke's cleft cysts are etiologically related.

We also found for the first time to our knowledge that these rare 'ciliated craniopharyngioma' cases were positive for the BRAF V600E mutation by genotyping and immunohistochemistry, confirming that these cases are likely more closely related to papillary craniopharyngioma than Rathke's cleft cysts with extensive squamous metaplasia, ${ }^{22}$ and that they may also respond favorably to pharmacologic inhibition of MAPK signaling as has been shown in two patients with papillary craniopharyngioma. ${ }^{31,32,46}$ Given the robust preservation of ciliated epithelium in a recurrent lesion after an extended period of time, it remains unclear whether 'ciliated papillary craniopharyngiomas' may represent 'transitional lesions', or a distinct stable phenotype of papillary craniopharyngioma. It is notable that these rare regions of ciliated epithelium were not readily noted during the initial analysis and histologic review of the specimens, only becoming clearly evident after FOXJ1 and/or cilial staining. We therefore expect that FOXJ1 and TUBA4A/ARL13B will greatly assist in future studies aimed at exploring the etiology of papillary craniopharyngioma and the 'ciliated papillary craniopharyngioma' phenotype.

In contrast to papillary craniopharyngioma, no regions of adjacent FOXJ1-positive ciliated epithelium were found in continuity with the neoplastic squamous epithelium in adamantinomatous craniopharyngioma. One case showed a separate fragment of ciliated epithelium that could be identified as sinonasal epithelium on the basis of glandular structures subjacent to the epithelium. It is important to consider the possibility of sinonasal 'contamination' and the route of resection/biopsy when evaluating limited tissue given that many small lesions of the sellar/suprasellar space are resected transnasally/transsphenoidally. Nevertheless, the lack of associated Rathke's cleft cyst-type ciliated epithelium in all cases provides additional support for the hypothesis that adamantinomatous craniopharyngiomas do not arise in association with Rathke's cleft cysts as hypothesized for papillary craniopharyngioma, but rather arise from other derivatives of the primitive stomodeal ectoderm.

We used ARL13B immunohistochemistry to show for the first time, to our knowledge, the widespread presence of primary cilia in both adamantinomatous craniopharyngioma and papillary craniopharyngioma. Primary cilia were limited to the basally oriented cells near fibrovascular cores in papillary craniopharyngioma in all the cases that we examined, whereas they were diffusely expressed by both basally oriented and more apically oriented cells in adamantinomatous craniopharyngioma, again in all the cases that we examined. Interestingly, the basal cells of adamantinomatous craniopharyngioma and papillary craniopharyngioma frequently showed a well-ordered architecture, with primary cilia extending from the apical ('anti-stromal') surface of the basal cells. The basis of this orientation is not known, but it suggests some preservation of individual cell polarity in the neoplastic epithelial cells. The diffuse and striking expression of primary cilia in adamantinomatous craniopharyngioma, observed in $100 \%$ of our cases by ARL13B staining and readily 
visible from low power, may additionally be used as an effective independent positive diagnostic feature of adamantinomatous craniopharyngioma compared to other lesions of the sellar region.

We hypothesized that the expression of primary cilia in adamantinomatous craniopharyngioma and papillary craniopharyngioma reflected the presence of active hedgehog signaling in these lesions. mRNA for $S H H$ and for downstream components of the hedgehog pathway, PTCH1 and GLI2, were strongly expressed in adamantinomatous craniopharyngioma cases. The hedgehog and Wnt/ $\beta$-catenin pathways have extensive cross-regulation. Hedgehog has long been known to directly regulate $\mathrm{Wnt} / \beta$-catenin signaling in development, ${ }^{47}$ and critical hedgehog pathway genes such as $S H H$ contain TCF/LEF binding promoter sequences in mice. ${ }^{48}$ Adamantinomatous craniopharyngiomas are genetically simple neoplasms with activating CTNNB1 mutations often representing the only identifiable frequently recurrent oncogenic driver event. ${ }^{15}$ Our identification in adamantinomatous craniopharyngioma of primary cilia throughout the epithelium in addition to widespread expression of well-known direct downstream transcriptional targets of activated hedgehog signaling (PTCH1 and GLI2) ${ }^{5}$ supports a model in which the focal expression of $S H H$ mRNA in cells with nuclear and cytoplasmic $\beta$-catenin translocation diffusely activates hedgehog signaling through paracrine cilia-dependent signaling mechanisms.

Given that hedgehog pathway activation by $\mathrm{SHH}$ requires primary cilia in vertebrates, the widespread distribution of cilia indicates continued responsiveness to the SHH ligand signal in adamantinomatous craniopharyngioma. Because coordinated strong expression of PTCH1 and GLI2 in association with diffuse ciliation suggests active and diffuse hedgehog signaling in the adamantinomatous craniopharyngioma epithelium, the identification of primary cilia in both this and other contexts by ARL13B immunohistochemistry may not only provide diagnostic information but may also suggest a distinct and actionable susceptibility to targeted therapies, ${ }^{32}$ such as hedgehog pathway inhibitors or compounds that target the ciliogenesis machinery. Though mutant $\beta$ catenin is apparently fundamental to adamantinomatous craniopharyngioma biology, there are as of yet no widely available therapeutic options for directly targeting this mutant protein, thus alternative strategies could be valuable.

In contrast to the widespread presence of primary cilia in adamantinomatous craniopharyngioma epithelial cells, primary cilia are limited in distribution to the first two to three layers of basally oriented cells surrounding the fibrovascular cores in papillary craniopharyngioma. Papillary craniopharyngiomas demonstrated diffuse low-level expression of PTCH1 and GLI2 compared with adamantinomatous craniopharyngioma, but no identifiable $S H H$ expression. Accordingly, it is likely that hedgehog pathway activity is low or absent in papillary craniopharyngioma despite the low-level presence of downstream pathway components. $\beta$-catenin is found localized to the membrane in all the cases of papillary craniopharyngioma that we examined, suggesting absence of active $\mathrm{Wnt} / \beta$-catenin signaling. The mechanisms underlying expression of primary cilia in the basal layers, and the significance of this finding for tumorigenesis, are therefore unclear and may not be related to hedgehog or Wnt/ $\beta$-catenin signaling. Nevertheless, the remarkably specific localization of primary cilia to the basal layers in all the cases suggests precise regulation of primary ciliogenesis in papillary craniopharyngioma cells, which may have significance for the underlying biology of these tumors.

Primary cilia are absent or attenuated in a proliferation-independent manner in many diverse cancers studied to date with the frequent notable exception of hedgehog and Wnt/ $\beta$-catenin-driven tumors. Multiple mechanisms have been proposed for their loss, including direct downregulation of ciliogenesis genes, activation of the cilial disassembly factors HDAC6 and AURKA, and loss of VHL. ${ }^{49}$ Loss of cilia may provide selective advantages to tumors as in the case of medulloblastomas harboring activating GLI2 mutations, where cilia loss abolishes counter-regulatory repressor GLI signal. ${ }^{50}$ Primary cilium disassembly is required for cell cycle progression in cells that have a normal complement of centrioles. ${ }^{11}$ Generation and disassembly of the cilium is energetically costly, and therefore downregulation of the primary cilia is a potential adaptive strategy to increase cell cycle efficiency in tumor cells that do not require cilial signaling. However, in cases where oncogenic signaling relies on primary cilia, as in the case of hedgehog-driven tumors, primary cilia must be elaborated by the tumor cells unless further events, such as GLI or RAS activation, abrogate that requirement.

In summary, we expect that FOXJ1 and ARL13B will be useful immunohistochemical markers for monitoring the presence of cells with motile and/or primary cilia in a wide variety of settings. Such markers may not only improve diagnostic accuracy, but may also provide useful insights into the biology of lesions, which may suggest novel therapeutic interventions. Characterization of the motile ciliogenesis program in ependymoma via FOXJ1 immunohistochemistry has already yielded interesting insights into disease biology. ${ }^{39}$ We expect that FOXJ1 and TUBA4A/ ARL13B immunohistochemistry will prove useful in the evaluation of other tissues. In particular, because ARL13B immunohistochemistry enables the robust characterization of primary cilia in routine formalinfixed paraffin-embedded sections, its use in a wide variety of tissues should provide important insights into the poorly understood biology of primary cilia and their relationship to neoplastic and non-neoplastic diseases in humans. 


\section{Acknowledgments}

We thank members of the Santagata lab for helpful discussions, Christopher Crum for advice on marker development, and Marian Slaney, Karen Bryan, and Sebastian Valentin for technical assistance with tissue processing. This study was supported by the Jared Branfman Sunflowers for Life Fund for Pediatric Brain and Spinal Cancer Research (SS) and the Ludwig Center at Harvard (SS). We thank Dana-Farber/Harvard Cancer Center in Boston, MA, USA, for the use of the Specialized Histopathology Core, which provided tissue imaging services. DanaFarber/Harvard Cancer Center is supported in part by an NCI Cancer Center Support Grant \# NIH 5 P30 CA06516.

\section{Disclosure/conflict of interest}

The authors declare no conflict of interest.

\section{References}

1 Sloboda RD. Primary Cilia. Elsevier/Academic Press: Amsterdam, The Netherlands, 2009, 408pp.

2 Tang TK. Centriole biogenesis in multiciliated cells. Nat Cell Biol 2013;15:1400-1402.

3 Cibas ES, Ducatman BS. Cytology: Diagnostic Principles and Clinical Correlates. Elsevier: Philadelphia, PA, USA, 2014, 576pp.

4 Shin JL, Asa SL, Woodhouse LJ, et al. Cystic lesions of the pituitary: clinicopathological featuresdistinguishing craniopharyngioma, Rathke's cleft cyst, and arachnoid cyst. J Clin Endocrinol Metab 1999;84:3972-3982.

5 Briscoe J, Thérond PP. The mechanisms of Hedgehog signalling and its roles in development and disease. Nat Rev Mol Cell Biol 2013;14:416-429.

6 Goetz SC, Anderson KV. The primary cilium: a signalling centre during vertebrate development. Nat Rev Genet 2010;11:331-344.

7 Eberhart C. Multiple cilia suppress tumour formation. Nat Cell Biol 2016;18:368-369.

8 Li L, Grausam KB, Wang J, et al. Sonic Hedgehog promotes proliferation of Notch-dependent monociliated choroid plexus tumour cells. Nat Cell Biol 2016;18:418-430.

9 Praetorius HA, Spring KR. Bending the MDCK cell primary cilium increases intracellular calcium. J Membr Biol 2001;184:71-79.

10 Delling M, Indzhykulian AA, Liu X, et al. Primary cilia are not calcium-responsive mechanosensors. Nature 2016;531:656-660.

11 Ishikawa H, Marshall WF. Ciliogenesis: building the cell's antenna. Nat Rev Mol Cell Biol 2011;12:222-234.

12 Hildebrandt F, Benzing T, Katsanis N. Ciliopathies. N Engl J Med 2011;364:1533-1543.

13 Snedecor ER, Sung CC, Moncayo A, et al. Loss of primary cilia in melanoma cells is likely independent of proliferation and cell cycle progression. J Invest Dermatol 2015;135:1456.

14 Menzl I, Lebeau L, Pandey R, et al. Loss of primary cilia occurs early in breast cancer development. Cilia 2014;3:7.
15 Brastianos PK, Taylor-Weiner A, Manley PE, et al. Exome sequencing identifies BRAF mutations in papillary craniopharyngiomas. Nat Genet 2014;46:161-165.

16 Pekmezci M, Louie J, Gupta N, et al. Clinicopathological characteristics of adamantinomatous and papillary craniopharyngiomas: University of California, San Francisco experience 1985-2005. Neurosurgery 2010;67:1341-1349.

17 Giangaspero F, Burger PC, Osborne DR, et al. Suprasellar papillary squamous epithelioma ("papillary craniopharyngioma"). Am J Surg Pathol 1984;8:57-64.

18 Crotty TB, Scheithauer BW, Young WF Jr. Papillary craniopharyngioma: a clinicopathological study of 48 cases. J Neurosurg 1995;83:206-214.

19 Louis DN, Ohgaki H, Wiestler OD, et al. WHO Classification of Tumours of the Central Nervous System. IARC: Lyon, France. 2007.

20 Larkin SJ, Preda V, Karavitaki N, et al. BRAF V600E mutations are characteristic for papillary craniopharyngioma and may coexist with CTNNB1-mutated adamantinomatous craniopharyngioma. Acta Neuropathol 2014;127:927-929.

21 Schweizer L, Capper D, Hölsken A, et al. BRAF V600E analysis for the differentiation of papillary craniopharyngiomas and Rathke's cleft cysts. Neuropathol Appl Neurobiol 2015;41:733-742.

$22 \mathrm{Kim} \mathrm{JH}$, Paulus W, Heim S. BRAF V600E mutation is a useful marker for differentiating Rathke's cleft cyst with squamous metaplasia from papillary craniopharyngioma. J Neurooncol 2015;123:189-191.

23 Marucci G, de Biase D, Zoli M, et al. Targeted BRAF and CTNNB1 next-generation sequencing allows proper classification of nonadenomatous lesions of the sellar region in samples with limiting amounts of lesional cells. Pituitary 2015;18:905-911.

24 Gomes DC, Jamra SA, Leal LF, et al. Sonic Hedgehog pathway is upregulated in adamantinomatous craniopharyngiomas. Eur J Endocrinol 2015;172:603-608.

25 Gump JM, Donson AM, Birks DK, et al. Identification of targets for rational pharmacological therapy in childhood craniopharyngioma. Acta Neuropathol Commun $2015 ; 3: 30$.

26 Andoniadou CL, Gaston-Massuet C, Reddy R, et al. Identification of novel pathways involved in the pathogenesis of human adamantinomatous craniopharyngioma. Acta Neuropathol 2012;124:259-271.

27 Bailey JM, Mohr AM, Hollingsworth MA. Sonic hedgehog paracrine signaling regulates metastasis and lymphangiogenesis in pancreatic cancer. Oncogene 2009; 28:3513-3525.

28 Seeley ES, Carrière C, Goetze T, et al. Pancreatic cancer and precursor pancreatic intraepithelial neoplasia lesions are devoid of primary cilia. Cancer Res 2009;69:422-430.

29 Tian H, Callahan CA, DuPree KJ, et al. Hedgehog signaling is restricted to the stromal compartment during pancreatic carcinogenesis. Proc Natl Acad Sci USA 2009; 106:4254-4259.

30 Jones RT, Abedalthagafi MS, Brahmandam M, et al. Cross-reactivity of the BRAF VE1 antibody with epitopes in axonemal dyneins leads to staining of cilia. Mod Pathol 2015;28:596-606.

31 Brastianos PK, Shankar GM, Gill CM, et al. Dramatic response of BRAF V600E mutant papillary craniopharyngioma to targeted therapy. J Natl Cancer Inst 2015;23:108.

32 Aylwin SJ, Bodi I, Beaney R. Pronounced response of papillary craniopharyngioma to treatment with vemurafenib, a BRAF inhibitor. Pituitary 2015; e-pub ahead of print. 
33 Schweizer L, Capper D, Hölsken A, et al. BRAF V600E analysis for the differentiation of papillary craniopharyngiomas and Rathke's cleft cysts. Neuropathol Appl Neurobiol 2015;41:733-742.

34 Xin W, Rubin MA, McKeever PE. Differential expression of cytokeratins 8 and 20 distinguishes craniopharyngioma from Rathke cleft cyst. Arch Pathol Lab Med 2002;126:1174-1178.

35 Buslei R, Hölsken A, Hofmann B, et al. Nuclear betacatenin accumulation associates with epithelial morphogenesis in craniopharyngiomas. Acta Neuropathol 2007;113:585-590.

36 Le BH, Towfighi J, Kapadia SB, et al. Comparative immunohistochemical assessment of craniopharyngioma and related lesions. Endocr Pathol 2007;18:23-30.

$37 \mathrm{Yu} \mathrm{X}, \mathrm{Ng} \mathrm{CP}$, Habacher $\mathrm{H}$, et al. Foxj1 transcription factors are master regulators of the motile ciliogenic program. Nat Genet 2008;40:1445-1453.

38 Borovina A, Superina S, Voskas D, et al. Vangl2 directs the posterior tilting and asymmetric localization of motile primary cilia. Nat Cell Biol 2012;12:407-412.

39 Abedalthagafi M, Wu MP, Merrill PH, et al. Decreased FOXJ1 expression and its ciliogenesis programme in aggressive ependymoma and choroid plexus tumours. J Pathol 2016;238:584-597.

$40 \mathrm{Du}$ Z, Abedalthagafi M, Aizer AA, et al. Increased expression of the immune modulatory molecule PD-L1 (CD274) in anaplastic meningioma. Oncotarget 2015;6:4704-4716.

41 Walentek P. Ciliary transcription factors in cancer how understanding ciliogenesis can promote detection and prognosis of cancer types. J Pathol 2016;239:6-9.
42 Rustici G, Kolesnikov N, Brandizi M, et al. ArrayExpress update-trends in database growth and links to data analysis tools. Nucleic Acids Res 2013;41: D987-D990.

$43 \mathrm{Lu} \mathrm{H}$, Toh MT, Narasimhan V, et al. A function for the Joubert syndrome protein Arl13b in ciliary membrane extension and ciliary length regulation. Dev Biol 2015; 397:225-236.

44 Okada T, Fujitsu K, Miyahara K, et al. Ciliated craniopharyngioma-case report and pathological study. Acta Neurochir (Wien) 2010;152:303-306.

45 Hölsken A, Sill M, Merkle J, et al. Adamantinomatous and papillary craniopharyngiomas are characterized by distinct epigenomic as well as mutational and transcriptomic profiles. Acta Neuropathol Commun 2016; $4: 20$.

46 Brastianos PK, Santagata S. Endocrine tumors: BRAF V600E mutations in papillary craniopharyngioma. Eur J Endocrinol 2016;174:R139-R144.

47 Gilbert SF. Developmental Biology, 10th edn. Sinauer Associates: Sunderland, MA, USA, 2013, 719pp.

48 Iwatsuki $\mathrm{K}$, Liu HX, Gronder A, et al. Wnt signaling interacts with Shh to regulate taste papilla development. Proc Natl Acad Sci USA 2007;104: 2253-2258.

49 Seeger-Nukpezah T, Little JL, Serzhanova V, et al. Cilia and cilia-associated proteins in cancer. Drug Discov Today Dis Mech 2013;10:e135-e142.

50 Han Y, Kim HJ, Dlugosz AA, et al. Dual and opposing roles of primary cilia in medulloblastoma development. Nat Med 2009;15:1062-1065.

Supplementary Information accompanies the paper on Modern Pathology website (http://www.nature.com/ modpathol) 